\title{
Computational estimate of the photophysical capabilities of four series of organometallic iron(II) complexes.
}

Isabelle M. Dixon, * Gauthier Boissard, Hannah Whyte, Fabienne Alary, Jean-Louis Heully. Laboratoire de Chimie et Physique Quantiques, UMR 5626 CNRS/Université Toulouse 3-Paul Sabatier, Toulouse, France.

\section{Contents}

Computational details p S2

Geometries : selected distances p S3

${ }^{5} \mathrm{MC}$ geometries p S6

Orbitals : Selected eigenvalues and energy gaps p S9

Excited states : Mulliken spin densities and spin contamination p S10

References p S11 


\section{Computational details}

For the model nickel complexes, geometries were optimized using Orca $2.8^{1}$ with the B3LYP functional, ${ }^{2}$ the ZORA $^{3}$ scalar relativity, the def2-TZVP basis set, ${ }^{4}$ and the VDW empirical dispersion correction. ${ }^{5}$ Geometry optimizations were followed by numerical frequency calculations.

For the iron complexes, lowest singlet, triplet, and quintet states were optimized using Orca 3 with the PBE0 functional, ${ }^{6}$ the ZORA scalar relativity, the def2-TZVP(-f) basis set (except for complexes 12, 17 and 21 which used the smaller def2-SVP basis set for SCF convergence issues) and the $\mathrm{D}^{\mathrm{B} J}{ }^{7}$ dispersion. Geometry optimizations were followed by numerical frequency calculations. Final single point energy calculations were undertaken using B3LYP*8/D3BJ/ZORA/def2-TZVP(-f) at the PBE0 equilibrium geometries, as discussed previously. ${ }^{9}$ A recent thorough study by Kepp, ${ }^{10}$ covering 30 iron complexes, showed that B3LYP* was the most accurate of 12 functionals with respect to experimental data, when dispersion and relativity are taken into account (i.e. same methodology as the one we use). In addition, as B3LYP* was initially conceived to describe the energetics of iron complexes containing heteroatoms such as phosphorus, ${ }^{8}$ this methodology should be reliable for the proposed series of compounds.

Force constants were computed using the orca_vib program. 
Geometries : Selected distances $(\AA)$.

The highlighted values are those that vary the most by comparison to the GS geometry.

Table S1 : $\mathrm{FeN}_{5} \mathrm{C}$ series

\begin{tabular}{l|l|l|l|l}
\multicolumn{1}{c|}{ complex } & & GS & 3MC & 5MC \\
\hline $\mathbf{1}$ & Fe-N1 & 1.967 & 2.223 & 2.246 \\
& Fe-N2 & 1.869 & 2.035 & 2.164 \\
& Fe-N3 & 1.981 & 2.223 & 2.227 \\
Fe-N4 & 1.993 & 1.991 & 2.221 \\
& Fe-N5 & 1.974 & 1.992 & 2.199 \\
& Fe-C & 1.908 & 1.922 & 2.057 \\
\hline $\mathbf{2}$ & Fe-N1 & 1.962 & 2.227 & 2.211 \\
& Fe-N2 & 1.862 & 2.013 & 2.169 \\
& Fe-N3 & 1.973 & 2.259 & 2.229 \\
& Fe-N4 & 1.993 & 1.999 & 2.257 \\
& Fe-N5 & 1.972 & 1.976 & 2.189 \\
& Fe-C & 1.906 & 1.915 & 2.021 \\
\hline $\mathbf{3}$ & Fe-N1 & 1.961 & 2.225 & 2.230 \\
& Fe-N2 & 1.863 & 2.015 & 2.161 \\
& Fe-N3 & 1.979 & 2.246 & 2.251 \\
& Fe-N4 & 1.992 & 2.000 & 2.240 \\
& Fe-N5 & 1.971 & 1.972 & 2.177 \\
& Fe-C & 1.906 & 1.910 & 2.007 \\
\hline $\mathbf{4}$ & Fe-N1 & 1.994 & 2.242 & 2.223 \\
& Fe-N2 & 1.882 & 2.045 & 2.148 \\
& Fe-N3 & 2.021 & 2.348 & 2.247 \\
& Fe-N4 & 2.008 & 2.007 & 2.235 \\
& Fe-N5 & 1.999 & 2.008 & 2.205 \\
& Fe-C & 2.033 & 2.018 & 2.176
\end{tabular}

$\mathrm{N} 1, \mathrm{~N} 2, \mathrm{~N} 3=$ tpy ; N4, N5=bpy (N4 is trans to the $\mathrm{C}$ atom)

Table S2 : $\mathrm{FeN}_{3} \mathrm{P}_{2} \mathrm{C}$ series

\begin{tabular}{l|l|l|l|l}
\multicolumn{1}{c|}{ complex } & & GS & 3MC & 5MC \\
\hline $\mathbf{5}$ & Fe-N1 & 2.021 & 2.276 & 2.251 \\
& Fe-N2 & 1.904 & 2.054 & 2.153 \\
& Fe-N3 & 2.015 & 2.272 & 2.229 \\
& Fe-P4 & 2.245 & 2.283 & 2.625 \\
& Fe-P5 & 2.232 & 2.245 & 2.503 \\
& Fe-C & 1.918 & 1.937 & 2.088 \\
\hline $\mathbf{6}$ & Fe-N1 & 2.015 & 2.282 & 2.259 \\
& Fe-N2 & 1.898 & 2.043 & 2.157 \\
& Fe-N3 & 2.009 & 2.273 & 2.233 \\
& Fe-P4 & 2.243 & 2.301 & 2.708 \\
& Fe-P5 & 2.219 & 2.225 & 2.476 \\
\hline $\mathbf{7}$ & Fe-C & 1.920 & 1.927 & 2.035 \\
\hline & Fe-N1 & 2.018 & 2.292 & 2.220 \\
& Fe-N2 & 1.892 & 2.034 & 2.183 \\
& Fe-N3 & 2.021 & 2.273 & 2.206 \\
& Fe-P4 & 2.250 & 2.305 & 3.079 \\
& Fe-P5 & 2.240 & 2.220 & 2.499 \\
& Fe-C & 1.916 & 1.920 & 2.014
\end{tabular}




\begin{tabular}{|c|c|c|c|c|}
\hline 8 & $\begin{array}{l}\text { Fe-N1 } \\
\text { Fe-N2 } \\
\text { Fe-N3 } \\
\text { Fe-P4 } \\
\text { Fe-P5 } \\
\text { Fe-C }\end{array}$ & $\begin{array}{l}2.039 \\
1.909 \\
2.036 \\
2.291 \\
2.261 \\
2.077\end{array}$ & $\begin{array}{l}2.340 \\
2.056 \\
2.283 \\
2.354 \\
2.260 \\
2.072\end{array}$ & \begin{tabular}{|l}
2.278 \\
2.154 \\
2.282 \\
2.677 \\
2.551 \\
2.215
\end{tabular} \\
\hline
\end{tabular}

$\mathrm{N} 1, \mathrm{~N} 2, \mathrm{~N} 3=$ tpy ; $\mathrm{P} 4, \mathrm{P} 5=\mathrm{dmpe}(\mathrm{P} 4$ is trans to the $\mathrm{C}$ atom)

Table S3 : $\mathrm{FeN}_{4} \mathrm{C}_{2}$ series

\begin{tabular}{l|l|l|l|l} 
complex & GS & $\begin{array}{l}\text { lowest } \\
\text { triplet } \\
\text { state }\end{array}$ & \\
\hline $\mathbf{9}$ & Fe-N1 & 1.945 & 2.319 & 2.227 \\
& Fe-N2 & 1.976 & 2.134 & 2.287 \\
& Fe-N3 & 1.949 & 2.172 & 2.220 \\
& Fe-N4 & 1.983 & 1.998 & 2.374 \\
& Fe-C5 & 1.914 & 1.918 & 2.088 \\
& Fe-C6 & 1.910 & 1.961 & 2.080 \\
\hline $\mathbf{1 0}$ & Fe-N1 & 1.936 & 1.946 & 2.226 \\
& Fe-N2 & 1.973 & 2.011 & 2.322 \\
& Fe-N3 & 1.942 & 1.949 & 2.228 \\
& Fe-N4 & 1.976 & 2.014 & 2.444 \\
& Fe-C5 & 1.910 & 1.894 & 2.058 \\
& Fe-C6 & 1.909 & 1.893 & 2.048 \\
\hline $\mathbf{1 1}$ & Fe-N1 & 1.949 & 1.949 & 2.242 \\
& Fe-N2 & 1.980 & 2.009 & 2.297 \\
& Fe-N3 & 1.965 & 1.959 & 2.292 \\
& Fe-N4 & 1.984 & 2.018 & 2.422 \\
& Fe-C5 & 1.902 & 1.886 & 2.050 \\
& Fe-C6 & 1.906 & 1.895 & 2.048 \\
\hline $\mathbf{1 3}$ & Fe-N1 & 1.935 & 1.952 & 2.138 \\
& Fe-N2 & 1.957 & 1.987 & 2.218 \\
& Fe-N3 & 1.965 & 1.973 & 2.376 \\
& Fe-N4 & 1.967 & 2.004 & 2.301 \\
& Fe-C5 & 1.906 & 1.896 & 2.038 \\
& Fe-C6 & 1.913 & 1.903 & 2.061 \\
\hline & Fe-N1 & 1.974 & 2.267 & 2.175 \\
& Fe-N2 & 2.006 & 2.151 & 2.216 \\
& Fe-N3 & 1.993 & 2.218 & 2.217 \\
& Fe-N4 & 2.029 & 2.027 & 2.269 \\
& Fe-C5 & 1.974 & 1.982 & 2.214 \\
& Fe-C6 & 1.991 & 2.063 & 2.228 \\
& Ftrans & & & \\
& & & \\
& &
\end{tabular}

$\mathrm{N} 2$ is trans to $\mathrm{C6}, \mathrm{P} 4$ is trans to $\mathrm{C} 5$.

Table $\mathrm{S4}: \mathrm{FeN}_{2} \mathrm{P}_{2} \mathrm{C}_{2}$ series

\begin{tabular}{l|l|l|l|l} 
complex & & GS & $\begin{array}{l}\text { lowest } \\
\text { triplet } \\
\text { state }\end{array}$ & 5MC \\
\hline $\mathbf{1 4}$ & Fe-N1 & 1.971 & 1.959 & 2.222 \\
& Fe-N2 & 1.991 & 1.987 & 2.264
\end{tabular}




\begin{tabular}{|c|c|c|c|c|}
\hline & $\begin{array}{l}\mathrm{Fe}-\mathrm{P} 3 \\
\mathrm{Fe}-\mathrm{P} 4 \\
\mathrm{Fe}-\mathrm{C} 5 \\
\mathrm{Fe}-\mathrm{C} 6\end{array}$ & $\begin{array}{l}2.210 \\
2.220 \\
1.921 \\
1.892 \\
\end{array}$ & $\begin{array}{l}2.253 \\
2.317 \\
1.919 \\
1.908 \\
\end{array}$ & $\begin{array}{l}2.491 \\
2.772 \\
2.083 \\
2.088 \\
\end{array}$ \\
\hline 15 & $\begin{array}{l}\text { Fe-N1 } \\
\text { Fe-N2 } \\
\text { Fe-P3 } \\
\text { Fe-P4 } \\
\text { Fe-C5 } \\
\text { Fe-C6 }\end{array}$ & $\begin{array}{l}1.968 \\
1.990 \\
2.196 \\
2.210 \\
1.923 \\
1.908\end{array}$ & $\begin{array}{l}1.971 \\
1.993 \\
2.222 \\
2.318 \\
1.902 \\
1.911\end{array}$ & $\begin{array}{l}2.245 \\
2.271 \\
2.480 \\
2.969 \\
2.047 \\
2.063\end{array}$ \\
\hline 16 & $\begin{array}{l}\text { Fe-N1 } \\
\text { Fe-N2 } \\
\text { Fe-P3 } \\
\text { Fe-P4 } \\
\text { Fe-C5 } \\
\text { Fe-C6 }\end{array}$ & $\begin{array}{l}1.967 \\
1.994 \\
2.199 \\
2.208 \\
1.913 \\
1.900 \\
\end{array}$ & $\begin{array}{l}1.975 \\
1.993 \\
2.229 \\
2.305 \\
1.892 \\
1.903 \\
\end{array}$ & $\begin{array}{l}2.282 \\
2.242 \\
2.502 \\
2.945 \\
2.047 \\
2.050 \\
\end{array}$ \\
\hline 17 & $\begin{array}{l}\text { Fe-N1 } \\
\text { Fe-N2 } \\
\text { Fe-P3 } \\
\text { Fe-P4 } \\
\text { Fe-C5 } \\
\text { Fe-C6 }\end{array}$ & $\begin{array}{l}1.973 \\
1.977 \\
2.198 \\
2.230 \\
1.903 \\
1.909\end{array}$ & $\begin{array}{l}1.972 \\
1.987 \\
2.234 \\
2.321 \\
1.901 \\
1.893\end{array}$ & $\begin{array}{l}2.208 \\
2.152 \\
2.575 \\
4.177 \\
2.006 \\
2.017\end{array}$ \\
\hline 18 & $\begin{array}{l}\text { Fe-N1 } \\
\text { Fe-N2 } \\
\text { Fe-P3 } \\
\text { Fe-P4 } \\
\text { Fe-C5 } \\
\text { Fe-C6 }\end{array}$ & $\begin{array}{l}1.996 \\
2.029 \\
2.305 \\
2.410 \\
2.019 \\
1.999\end{array}$ & $\begin{array}{l}2.173 \\
1.999 \\
3.616 \\
2.371 \\
2.029 \\
1.983\end{array}$ & $\begin{array}{l}2.243 \\
2.217 \\
2.591 \\
2.867 \\
2.210 \\
2.192\end{array}$ \\
\hline 19 & $\begin{array}{l}\text { Fe-N1 } \\
\text { Fe-N2 } \\
\text { Fe-P3 } \\
\text { Fe-P4 } \\
\text { Fe-C5 } \\
\text { Fe-C6 }\end{array}$ & $\begin{array}{l}1.980 \\
2.022 \\
2.214 \\
2.229 \\
1.920 \\
1.909 \\
\end{array}$ & $\begin{array}{l}1.970 \\
1.979 \\
2.279 \\
2.380 \\
1.897 \\
1.907 \\
\end{array}$ & $\begin{array}{l}2.278 \\
2.259 \\
2.565 \\
2.855 \\
2.043 \\
2.058 \\
\end{array}$ \\
\hline 20 & $\begin{array}{l}\text { Fe-N1 } \\
\text { Fe-N2 } \\
\text { Fe-P3 } \\
\text { Fe-P4 } \\
\text { Fe-C5 } \\
\text { Fe-C6 }\end{array}$ & $\begin{array}{l}1.982 \\
2.049 \\
2.261 \\
2.276 \\
1.919 \\
1.900\end{array}$ & $\begin{array}{l}1.976 \\
2.013 \\
2.301 \\
2.422 \\
1.898 \\
1.916\end{array}$ & $\begin{array}{l}2.264 \\
2.546 \\
2.641 \\
2.620 \\
2.054 \\
2.068\end{array}$ \\
\hline 21 & $\begin{array}{l}\text { Fe-N1 } \\
\text { Fe-N2 } \\
\text { Fe-P3 } \\
\text { Fe-P4 } \\
\text { Fe-C5 } \\
\text { Fe-C6 }\end{array}$ & $\begin{array}{l}1.990 \\
2.030 \\
2.259 \\
2.290 \\
1.909 \\
1.901\end{array}$ & $\begin{array}{l}1.970 \\
2.014 \\
2.359 \\
2.429 \\
1.890 \\
1.904\end{array}$ & $\begin{array}{l}2.251 \\
2.437 \\
2.675 \\
2.620 \\
2.035 \\
2.061\end{array}$ \\
\hline
\end{tabular}

$\mathrm{N} 2$ is trans to $\mathrm{C6}, \mathrm{P} 4$ is trans to $\mathrm{C} 5$. 
Figure S1 : ${ }^{5} \mathrm{MC}$ geometries

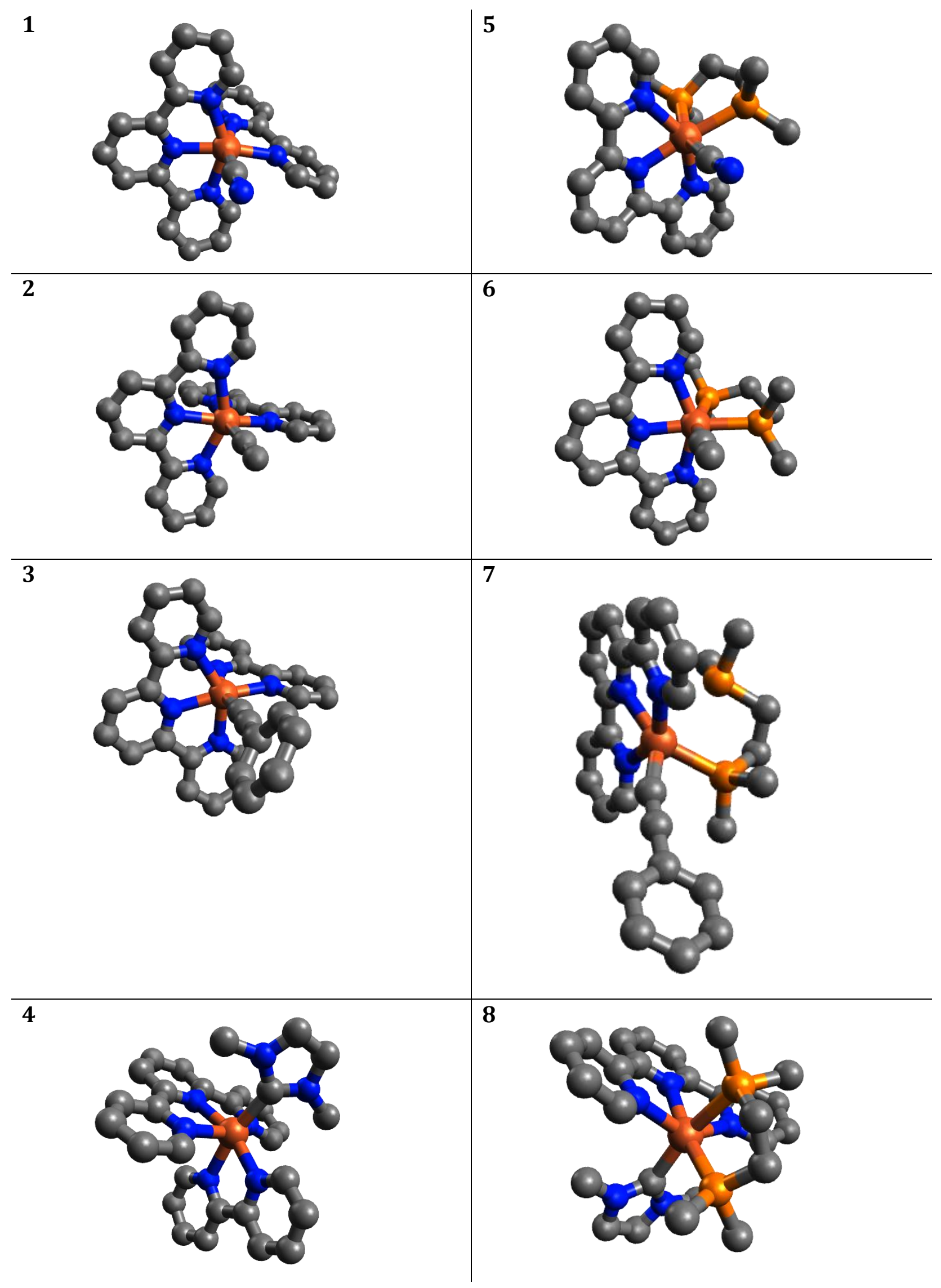




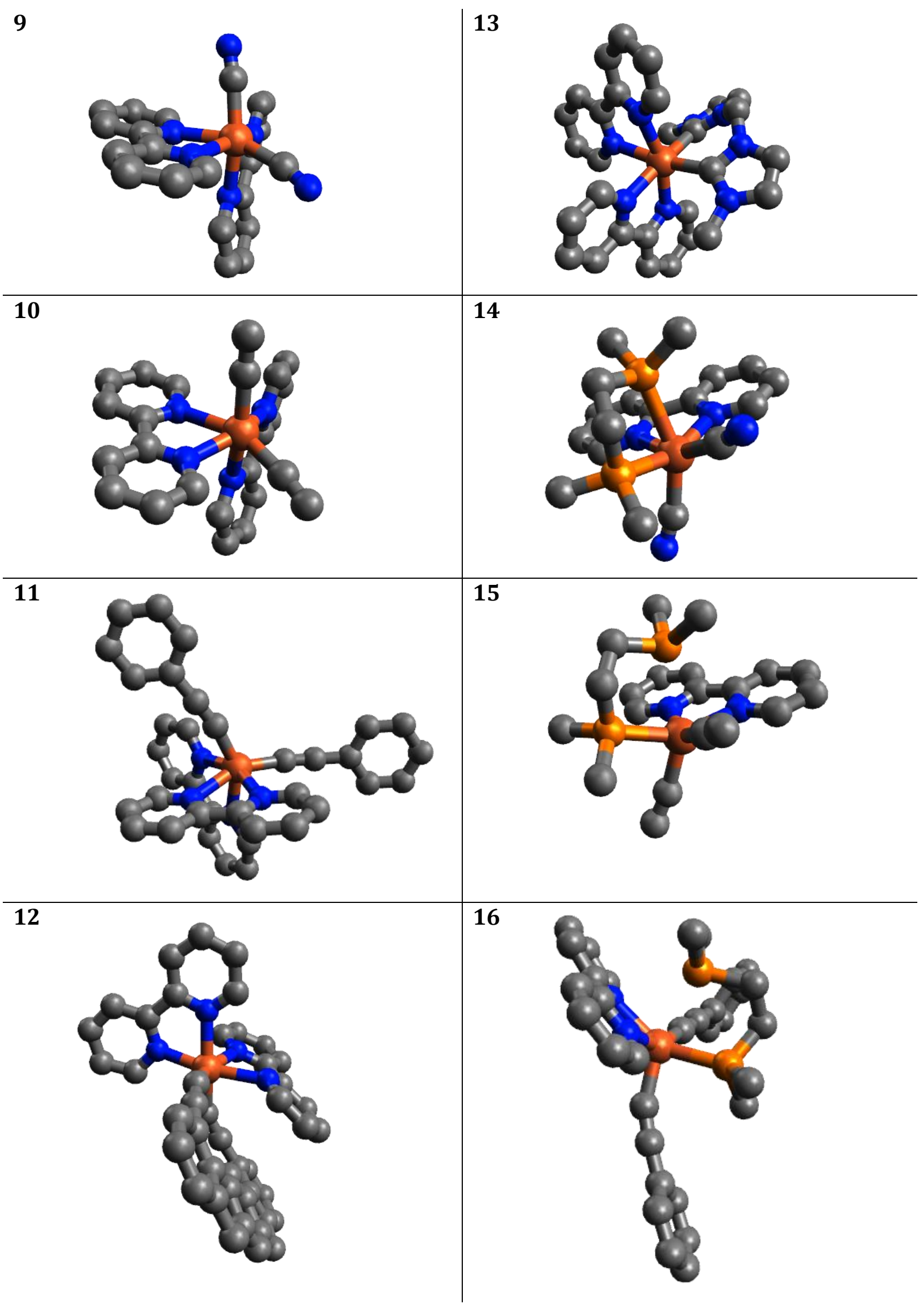




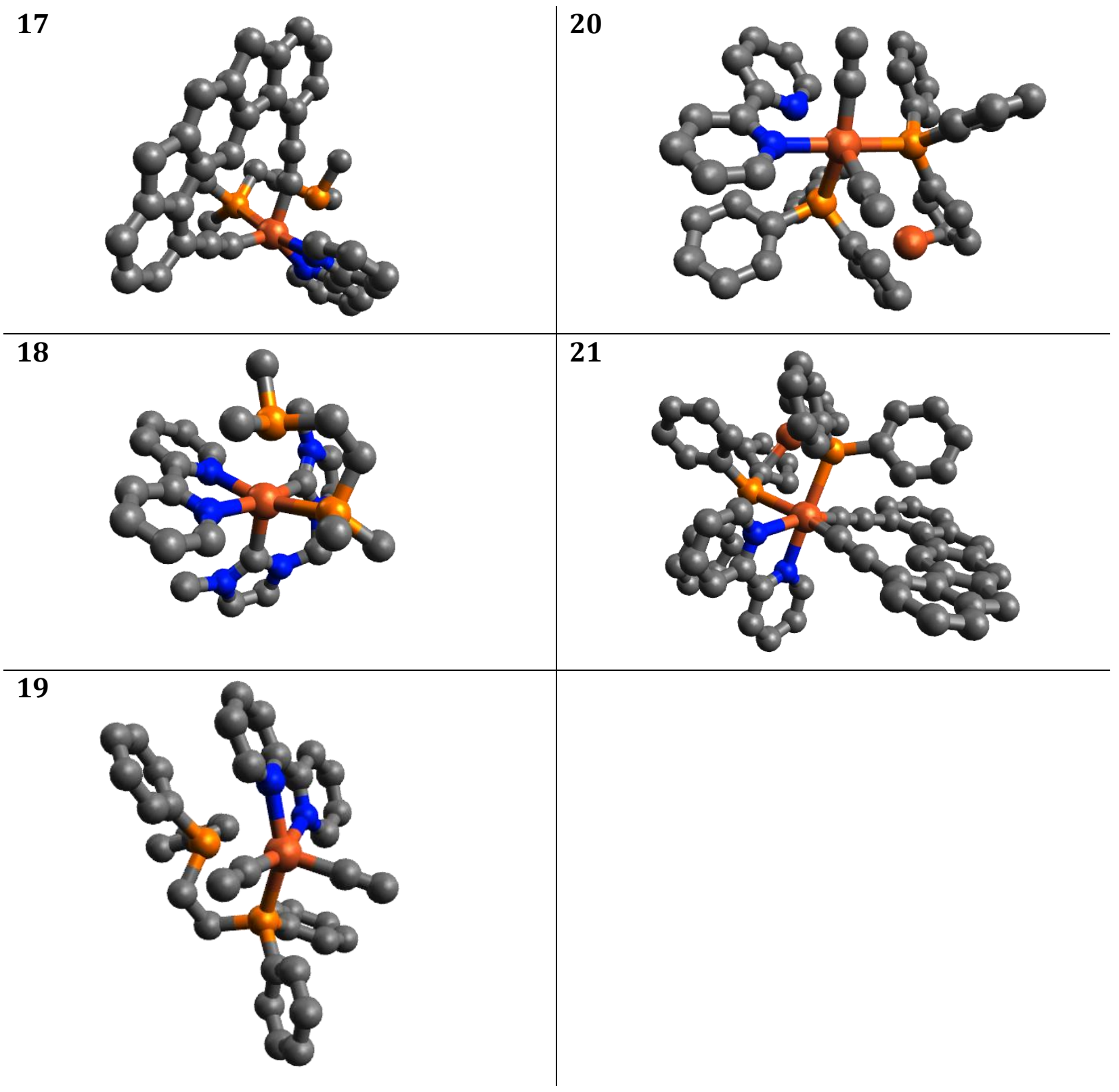


Table S5 : Orbitals : Selected eigenvalues and energy gaps (eV)

\begin{tabular}{|c|c|c|c|c|c|c|}
\hline $\mathrm{FeN}_{5} \mathrm{C}$ series & HO & $\mathbf{L U}$ & $d \sigma^{*}$ & HO-LU & HO-d $\sigma^{*}$ & LU-d $\sigma^{*}$ \\
\hline 1 & -7.99 & -5.24 & $\begin{array}{l}-2.94 \\
(\mathrm{LU}+9)\end{array}$ & 2.75 & 5.05 & 2.30 \\
\hline 2 & -7.49 & -5.06 & $\begin{array}{l}-2.67 \\
(L U+9)\end{array}$ & 2.43 & 4.82 & 2.39 \\
\hline 3 & -7.02 & -5.05 & $\begin{array}{l}-2.68 \\
(L U+9)\end{array}$ & 1.97 & 4.34 & 2.37 \\
\hline 4 & -10.74 & -7.88 & $\begin{array}{l}-5.87 \\
(L U+8)\end{array}$ & 2.86 & 4.87 & 2.01 \\
\hline $\mathrm{FeN}_{3} \mathrm{P}_{2} \mathrm{C}$ series & HO & LU & d $\sigma^{*}$ & HO-LU & HO-d $\sigma^{*}$ & LU-d $\sigma^{*}$ \\
\hline 5 & -8.19 & -5.23 & $\begin{array}{l}-3.42 \\
(L U+5)\end{array}$ & 2.96 & 4.77 & 1.81 \\
\hline 6 & -7.68 & -5.01 & $\begin{array}{l}-3.16 \\
(L U+5)\end{array}$ & 2.67 & 4.52 & 1.85 \\
\hline 7 & -7.19 & -5.02 & $\begin{array}{l}-3.17 \\
(L U+5)\end{array}$ & 2.17 & 4.02 & 1.85 \\
\hline 8 & -10.94 & -7.94 & $\begin{array}{l}-6.32 \\
(L U+4)\end{array}$ & 3.00 & 4.62 & 1.62 \\
\hline $\mathrm{FeN}_{4} \mathrm{C}_{2}$ series & HO & LU & $d \sigma^{*}$ & HO-LU & HO-d $\sigma^{*}$ & LU-d $\sigma^{*}$ \\
\hline 9 & -4.80 & -2.50 & $\begin{array}{l}+0.20 \\
(\mathrm{LU}+8)\end{array}$ & 2.30 & 5.00 & 2.70 \\
\hline 10 & -4.05 & -2.14 & $\begin{array}{l}+0.65 \\
(\mathrm{LU}+8)\end{array}$ & 1.91 & 4.70 & 2.79 \\
\hline 11 & -4.21 & -2.31 & $\begin{array}{l}+0.44 \\
(\mathrm{LU}+12)\end{array}$ & 1.90 & 4.65 & 2.75 \\
\hline 12 & -4.00 & -2.20 & $\begin{array}{l}+0.67 \\
(L U+12)\end{array}$ & 1.80 & 4.67 & 2.87 \\
\hline 13 & -10.55 & -7.71 & $\begin{array}{l}-5.60 \\
(L U+7)\end{array}$ & 2.84 & 4.95 & 2.11 \\
\hline $\mathrm{FeN}_{2} \mathrm{P}_{2} \mathrm{C}_{2}$ series & HO & LU & $d \sigma^{*}$ & HO-LU & HO-d $\sigma^{*}$ & LU-d $\sigma^{*}$ \\
\hline 14 & -4.92 & -2.43 & $\begin{array}{l}-0.09 \\
(L U+4)\end{array}$ & 2.49 & 4.83 & 2.34 \\
\hline 15 & -4.14 & -2.04 & $\begin{array}{l}+0.46 \\
(\mathrm{LU}+5)\end{array}$ & 2.10 & 4.87 & 2.50 \\
\hline 16 & -4.20 & -2.20 & $\begin{array}{l}+0.33 \\
(L U+9)\end{array}$ & 2.00 & 4.53 & 2.53 \\
\hline 17 & -4.06 & -2.11 & $\begin{array}{l}+0.60 \\
(\mathrm{LU}+9)\end{array}$ & 1.95 & 4.66 & 2.71 \\
\hline 18 & -10.73 & -7.84 & $\begin{array}{l}-6.09 \\
(L U+3)\end{array}$ & 2.89 & 4.64 & 1.75 \\
\hline 19 & -4.25 & -2.02 & $\begin{array}{l}+0.58 \\
(\mathrm{LU}+13)\end{array}$ & 2.23 & 4.83 & 2.60 \\
\hline 20 & -4.31 & -2.02 & $\begin{array}{l}+0.42 \\
(\mathrm{LU}+14)\end{array}$ & 2.29 & 4.73 & 2.44 \\
\hline 21 & -4.15 & -2.10 & $\begin{array}{l}+0.44 \\
(\mathrm{LU}+18)\end{array}$ & 2.05 & 4.59 & 2.54 \\
\hline
\end{tabular}


Table S6 : Excited states : Mulliken spin densities on iron and spin contamination (from the B3LYP* single point energy calculations)

\begin{tabular}{|c|c|c|c|c|}
\hline \multirow[t]{2}{*}{$\mathrm{FeN}_{5} \mathrm{C}$ series } & \multicolumn{2}{|c|}{ lowest triplet state $\left({ }^{3} \mathrm{MC}\right)$} & \multicolumn{2}{|c|}{ lowest quintet state $\left({ }^{5} \mathrm{MC}\right)$} \\
\hline & spin density & $\mathrm{S}^{2}$ & spin density & $\mathrm{S}^{2}$ \\
\hline 1 & 2.10 & 2.07 & 3.79 & 6.05 \\
\hline 2 & 2.16 & 2.09 & 3.81 & 6.06 \\
\hline 3 & 2.17 & 2.10 & 3.82 & 6.08 \\
\hline 4 & 2.08 & 2.04 & 3.79 & 6.04 \\
\hline \multirow[t]{2}{*}{$\mathrm{FeN}_{3} \mathrm{P}_{2} \mathrm{C}$ series } & \multicolumn{2}{|c|}{ lowest triplet state $\left({ }^{3} \mathrm{MC}\right)$} & \multicolumn{2}{|c|}{ lowest quintet state ( $\left.{ }^{5} \mathrm{MC}\right)$} \\
\hline & spin density & $\mathrm{S}^{2}$ & spin density & $\mathrm{S}^{2}$ \\
\hline 5 & 2.12 & 2.05 & 3.64 & 6.02 \\
\hline 6 & 2.14 & 2.06 & 3.64 & 6.03 \\
\hline 7 & 2.14 & 2.07 & 3.69 & 6.05 \\
\hline 8 & 2.15 & 2.05 & 3.69 & 6.02 \\
\hline \multirow[t]{2}{*}{$\mathrm{FeN}_{4} \mathrm{C}_{2}$ series } & \multicolumn{2}{|c|}{ lowest triplet state } & \multicolumn{2}{|c|}{ lowest quintet state ( $\left.{ }^{5} \mathrm{MC}\right)$} \\
\hline & spin density & $\mathrm{S}^{2}$ & spin density & $\mathrm{S}^{2}$ \\
\hline 9 & 2.13 & 2.08 & 3.82 & 6.06 \\
\hline 10 & 1.09 & 2.02 & 3.87 & 6.11 \\
\hline 11 & 1.07 & 2.02 & 3.88 & 6.12 \\
\hline 12 & 1.05 & 2.02 & 3.86 & 6.15 \\
\hline 13 & 2.08 & 2.04 & 3.77 & 6.04 \\
\hline \multirow[t]{2}{*}{$\mathrm{FeN}_{2} \mathrm{P}_{2} \mathrm{C}_{2}$ series } & \multicolumn{2}{|c|}{ lowest triplet state } & \multicolumn{2}{|c|}{ lowest quintet state ( $\left.{ }^{5} \mathrm{MC}\right)$} \\
\hline & spin density & $\mathrm{S}^{2}$ & spin density & $\mathrm{S}^{2}$ \\
\hline 14 & 1.08 & 2.02 & 3.70 & 6.04 \\
\hline 15 & 1.06 & 2.02 & 3.74 & 6.06 \\
\hline 16 & 1.05 & 2.02 & 3.74 & 6.07 \\
\hline 17 & 1.05 & 2.03 & 3.78 & 6.06 \\
\hline 18 & 2.11 & 2.04 & 3.73 & 6.02 \\
\hline 19 & 1.10 & 2.02 & 3.73 & 6.05 \\
\hline 20 & 1.12 & 2.03 & 3.74 & 6.04 \\
\hline 21 & 1.08 & 2.03 & 3.74 & 6.06 \\
\hline
\end{tabular}




\section{References}

(1) F. Neese, WIREs Comput. Mol. Sci. 2012, 2, 73-78.

(2) A. D. Becke, J. Chem. Phys. 1993, 98, 5648-5652 ; C. Lee, W. Yang, R. G. Parr, Phys. Rev. B 1988, 37, 785-789.

(3) C. J. van Wüllen, Chem. Phys. 1998, 109, 392-399.

(4) D. A. Pantazis, X. Y. Chen, C. R. Landis, F. Neese, J. Chem. Theory Comput. 2008, 4, 908919.

(5) S. Grimme, J. Comput. Chem. 2006, 27, 1787-1799.

(6) C. Adamo, V. Barone, J. Chem. Phys. 1999, 110, 6158-6170.

(7) S. Grimme, S. Ehrlich, L. Goerigk, J. Comput. Chem. 2011, 32, 1456-1465 ; S. Grimme, J. Antony, S. Ehrlich, H.Krieg, J. Chem. Phys. 2010, 132, 154104.

(8) M. Reiher, O. Salomon, B. A. Hess, Theor. Chem. Acc. 2001, 107, 48-55 ; M. Reiher, Inorg. Chem. 2002, 41, 6928-6935.

(9) I. M. Dixon, S. Khan, F. Alary, M. Boggio-Pasqua, J.-L. Heully, Dalton Trans. 2014, 43, 15898-15905.

(10) K.P. Kepp, Inorg. Chem. 2016, 55, 2717-2727. 